\title{
The Impact of Exacerbation Frequency on Clinical and Economic Outcomes in Swedish COPD Patients: The ARCTIC Study
}

This article was published in the following Dove Press journal: International Journal of Chronic Obstructive Pulmonary Disease

\author{
Kjell Larsson' \\ Christer Janson $\mathbb{D D}^{2}$ \\ Karin Lisspers $\mathbb{( D}^{3}$ \\ Björn Ställberg $\mathbb{D}^{3}$ \\ Gunnar Johansson (D) $^{3}$ \\ Florian S Gutzwiller (D) \\ Karen Mezzi ${ }^{4}$ \\ Bine Kjoeller Bjerregaard ${ }^{5}$ \\ Leif Jorgensen ${ }^{5}$ \\ 'Integrative Toxicology, The National \\ Institute of Environmental Medicine, \\ Karolinska Institute, Stockholm, Sweden; \\ ${ }^{2}$ Department of Medical Sciences: \\ Respiratory, Allergy and Sleep Research, \\ Uppsala University, Uppsala, Sweden; \\ ${ }^{3}$ Department of Public Health and Caring \\ Sciences, Family Medicine and Preventive \\ Medicine, Uppsala University, Uppsala, \\ Sweden; ${ }^{4}$ Novartis Pharma AG, Global \\ Patient Access, Basel, Switzerland; \\ ${ }^{5}$ IQVIA Solutions, Real World Evidence \\ Solutions, Copenhagen, Denmark
}

Correspondence: Kjell Larsson Integrative Toxicology, The National Institute of Environmental Medicine, Karolinska Institute, Stockholm, SE-17I 77, Sweden

Tel +46705820763

Email kjell.larsson@ki.se
Purpose: The aim of this study was to assess the association between exacerbation frequency and clinical and economic outcomes in patients with COPD.

Patients and Methods: Electronic medical record data linked to National Health Registries were collected from COPD patients at 52 Swedish primary care centers (2000-2014). The outcomes analyzed were exacerbation rate, mortality, COPD treatments, lung function and healthcare costs during the follow-up period. Based on the exacerbation rate two years before index date, the patients were initially classified into three groups, either 0,1 or $\geq 2$ exacerbations per year. After the index date, the classification into exacerbation groups was updated each year based on the exacerbation rate during the last year of follow-up. A sensitivity analysis was conducted excluding patients with asthma diagnosis from the analysis.

Results: In total 18,586 COPD patients were analyzed. A majority of the patients $(60-70 \%)$ who either have had no exacerbation or frequent exacerbations $(\geq 2 /$ year) during the preindex period remained in their group (ie, with 0 or $\geq 2$ annual exacerbations) during up to 11 years of follow-up. Compared with having no exacerbation, mortality was higher in patients having 1 (HR; 2.06 [1.93-2.20]) and $\geq 2$ (4.58 [4.33-4.84]) exacerbations at any time during the follow-up. Lung function decline was more rapid in patients with frequent exacerbations and there was an almost linear relationship between exacerbations frequency and mortality. Total healthcare costs were higher in the frequent exacerbation group ( $\geq 2 /$ year) than in patients with no or one exacerbation annually ( $<<0.0001$ for both). The results did not differ from the main analysis after exclusion of patients with a concurrent asthma diagnosis.

Conclusion: In addition to faster lung function decline and increased mortality, frequent exacerbations in COPD patients imply a significant economic burden.

Keywords: chronic obstructive pulmonary disease, exacerbations, mortality, lung function, healthcare cost, Sweden

\section{Introduction}

Chronic obstructive pulmonary disease (COPD) is a progressive disorder characterized by persistent respiratory symptoms and airflow limitation. It is a major cause of chronic morbidity and the third leading cause of death worldwide. ${ }^{1}$

COPD exacerbations are defined as an acute worsening of respiratory symptoms that results in additional therapy. ${ }^{2}$ Prevention of exacerbations is one of the significant components in the management of COPD as they negatively impact health status, and can result in hospitalization and re-admission, disease progression, rapid lung function decline and mortality. ${ }^{2}$ Annual rate of exacerbations varies among patients. Exacerbations become more frequent and serious with increasing 
disease severity ${ }^{3,4}$ but there is also clear evidence that certain patients have more frequent exacerbations irrespective of disease severity. ${ }^{4}$ Furthermore, economic studies have demonstrated that costs increase substantially with COPD severity, and a significant economic burden of COPD can be attributed to exacerbations. ${ }^{5-9}$

In previous studies, the quantitative relationship between exacerbation frequency and the impact on different health outcomes and mortality have rarely been assessed. Thus, it is not clear whether the increased risk of different health outcomes such as, quality of life, lung function decline and mortality caused by exacerbations, is associated with the fact that the patient experience exacerbations, no matter how often, or whether there is a quantitative association between the risk and the number of exacerbations. In the present study, we hypothesize that negative clinical effect caused by exacerbations are related to the frequency of exacerbations and not only to the tendency to have or not have exacerbations. It is therefore important to generate real-world evidence to better understand the effect of exacerbations on COPD. In Sweden, most patients with COPD are managed in primary care. The aim of this longitudinal study was to provide realworld evidence on the clinical and economic impact of exacerbation frequency among COPD patients in the Swedish primary care setting and to, more in detail, describe the relationship of exacerbations and clinical outcomes and economic consequences in COPD using a dynamic approach to exacerbations that resembles the clinical practice.

\section{Patients and Methods Study Design}

ARCTIC is a large, real-world, retrospective, Swedish cohort study conducted in 18,586 eligible, primary care, COPD patients. The study was conducted in accordance with the principles of the Declaration of Helsinki. Ethics approval was obtained from the local Ethical Regional Board in Uppsala, Sweden, on 11 December 2014 (number: 2014-397), for accessing the National Health Register and for recruiting primary care centers to the study. An amendment specifying additional analysis was approved by the Ethical Regional Board in Uppsala on 6 October 2017. Data from all records were de-identified, and therefore, the Ethics committee did not require patient consent. Electronic medical record (EMR) data were collected from patients with physician-diagnosed COPD in 52 primary care centers across Sweden between the years 2000-2014 using an established software system (Customized eXtraction Program, CXP 3.0). Collected data included age, gender, prescriptions (according to the World Health Organization Anatomic Therapeutic Chemical [ATC] codes), doctor's diagnoses (according to the International Classification of Diseases, tenth revision [ICD-10] codes), spirometry measurements $\left(\mathrm{FEV}_{1}\right.$ and FVC values), laboratory tests, healthcare professional visits and referrals. The centers covered urban and rural sites of varying sizes across Sweden. EMR data were linked by the Swedish National Board of Health and Welfare using individual patient identification (ID) numbers to National Registry data sources (patient IDs were pseudonymized). These data sources included: (i) the Longitudinal Integration Database for Health Insurance and Labour Market Studies (LISA), ${ }^{10}$ which contains sociodemographic data, including educational level, marital status and family situation, occupational status, retirement and economic compensation and social benefits; (ii) the National Patient Register, ${ }^{8}$ which contains data related to diagnosis from secondary care (ICD-10 code and associated position), including gender, age, region, hospital visits, specialty visits, hospital admissions and discharges, medical procedures and surgeries performed in inpatient and outpatient specialist settings; (iii) the National Prescription Register ${ }^{8}$ (from 2005), which tracks full details of all medications dispensed from community pharmacies' (ATC codes), including brand name, prescription date, dose, strength, pack size, specialty of the prescriber and costs associated with the drug prescription; and (iv) the Cause of Death Register, ${ }^{8}$ which is used to collect information of the deceased such as sex, date of death and the underlying cause of death.

\section{Study Patients}

The study population consisted of patients aged $\geq 40$ years who had received a physician's diagnosis of COPD (ICD10 code: J44), in a primary care setting (EMR database) or in a hospital setting (according to the National Patient Register). Patients' diagnoses were defined by ICD codes, while lung function was used to assess the degree of airflow limitation based on data collected from EMRs. Comorbidities were defined by recorded ICD10 diagnosis from primary and secondary care (both inpatients and outpatients). The study index date constituted the time of the first recorded physician's diagnosis of COPD during the enrolment timeframe. 


\section{Outcomes}

The outcomes analyzed were exacerbation rate, mortality, proportion of patients using various COPD treatments, change in lung function $\left(\mathrm{FEV}_{1}\right)$ and healthcare costs. Based on the exacerbation rate two years before index date (exacerbations defined as during follow-up), the patients were initially classified into three groups, either 0,1 or $\geq 2$ exacerbations per year. Exacerbation rate was assessed during the follow-up period. After the index date, the classification into exacerbation groups was updated each full year based on the exacerbation rate during the last year of follow-up. Acute exacerbations is defined as COPD-related hospitalizations (J44 in primary position or J44.0/J44.1 in secondary), emergency visits (J44.0/J44.1 in outpatient hospital care), or collection of oral steroids (ATC H02AB) or antibiotics targeted at respiratory diseases (ATC J01AA/J01CA) prescribed by either doctors in primary or secondary care. Exacerbations occurring within 14 days were considered as one single event. No separate analyses of exacerbations of different severity was performed.

Total survival was calculated based on the average exacerbations per year (ie, 0.1 and $\geq 2$ ) during a follow-up of up to 15 years (before death). For each year the collection of drugs was calculated and the cumulative dispensation of drugs for COPD during a follow-up period of 10 years (2006-2015) was assessed. For calculation of lung function $\left(\mathrm{FEV}_{1}\right)$ decline over time, $\geq 3$ lung function measurements after index date were required. Each lung function measurement has been assessed from a qualitative aspect to avoid incorrectly conducted measurements. The assessment was done without knowledge about the exacerbation rate. Lung function $\left(\mathrm{FEV}_{1}\right.$ and FVC) was measured after bronchodilatation and expressed as percent of predicted normal value and lung function decline was analyzed as annual $\mathrm{FEV}_{1}$ loss. Data were reported for patients who had experienced, on average, 0,1 , or $\geq 2$ exacerbations per year during follow-up. The direct healthcare costs were calculated for each year, from two years prior to index date and during follow-up for each of the groups. Differences in total costs were evaluated by fitting a log-linked gamma marginal model using generalized estimating equations (GEE) to the longitudinal data. Total costs excluding drug costs and separate data for drug costs was reported for patients with 0,1 and $\geq 2$ exacerbations per year during follow-up.

\section{Statistical Analysis}

A sample size calculation conducted prior to the study indicated that 13,800 patients were required to detect a $4 \%$ difference in exacerbation rate between groups, with a power of $80 \%$ and an alpha level of 5\% in the two-tailed test. The power calculation was based on finding a difference in rate of exacerbations between treatment groups. This calculation is also valid for any rate difference in the study. The statistical analysis software used was "PC SAS for Windows" Version 9.4. Patient demographics were described for COPD patients and stratified by annual exacerbation rate two years prior to the index date $(0,1$ and $\geq 2)$. The chi-square test was used to compare the two (or more if the analysis was stratified) groups of patients with the outcome (eg, percentage of patients with COPD and comorbidities) as the dependent variable and the group variable gender as the independent variable. As the classification of groups based on the exacerbation frequency were dynamic with yearly re-classifications, a Cox proportional hazards model with time-dependent frequency of exacerbations as a covariate was fitted. The frequency was calculated as the number of exacerbations in the previous 365 days. Frequency was updated continuously. To further investigate the relationship between exacerbations and mortality, the percent of patients who died was calculated, using a stepwise increase in the exacerbation rate of 0.2 exacerbations per year. The patients were censored at the last known time alive or at the end of the study. The analyses were presented as hazard ratios with $95 \%$ confidence interval (CI) for the group and covariate. An analysis of covariance was used to compare the difference between the groups with respect to hospital visits and primary care visits, number of prescriptions, and time from COPD diagnosis to the index date with age as covariate. Comorbidities were calculated at different time points as frequencies of the total study population at that time point. The overall comorbidity burden was assessed using the Charlson comorbidity index. A sensitivity analysis was conducted excluding patients with an asthma diagnosis to examine the impact of concurrent asthma on exacerbation rate, mortality and lung function decline.

\section{Results}

Out of 202,397 patients identified from the electronic medical records (EMR), 18,586 (53.8\% women) with COPD were identified as eligible for inclusion in the study. Baseline characteristics such as age, gender, comorbidities, treatment and health care utilization differed significantly between the groups having 0,1 or $\geq 2$ exacerbations per year the two years prior to index date (Table 1). Lung function values were recorded either before, at or after index date. The closest value to index date is given in Table 2. Baseline characteristics and lung function measurements of COPD patients without a concurrent asthma diagnosis has been reported in 
Table I Baseline Characteristics of 18,586 Patients with COPD Stratified by Exacerbation Rate

\begin{tabular}{|c|c|c|c|c|}
\hline \multirow[t]{3}{*}{ Variable } & \multicolumn{3}{|c|}{ Annual Exacerbation Rate 2 Years Prior to Index Date } & \multirow[t]{3}{*}{ P-value } \\
\hline & $\mathbf{0}$ & $\mathbf{I}$ & $\geq 2$ & \\
\hline & $\mathrm{N}=8092(43.5 \%)$ & $\mathrm{N}=4093(22.0 \%)$ & $\mathrm{N}=640 \mathrm{I}(34.5 \%)$ & \\
\hline Age (years) & 68.2 & 69.0 & 69.5 & $<0.0001$ \\
\hline Female & $4 \mid 25(5 \mid .0)$ & $2196(53.6)$ & $3672(57.4)$ & $<0.0001$ \\
\hline \multicolumn{5}{|l|}{ Comorbidity } \\
\hline Charlson Comorbidity Index value & 1.6 & 1.7 & 1.9 & $<0.0001$ \\
\hline Asthma, J45 & $944(11.7)$ & $564(13.8)$ & $1158(18.1)$ & $<0.0001$ \\
\hline Cardiovascular Disease, 100-199 & $2877(35.6)$ & $1549(37.8)$ & $2666(41.6)$ & $<0.0001$ \\
\hline Hypertension, 110 & $1660(20.5)$ & $898(21.9)$ & $1523(23.8)$ & $<0.0001$ \\
\hline Heart failure, 150 & $582(7.2)$ & $335(8.2)$ & $615(9.6)$ & $<0.0001$ \\
\hline Ischemic heart diseases, |20-125 & $760(9.4)$ & $425(10.4)$ & $730(11.4)$ & $<0.0001$ \\
\hline Cerebrovascular diseases, 160-169 & $317(3.9)$ & $186(4.5)$ & $272(4.2)$ & 0.2865 \\
\hline Diabetes Type I, EIO & $119(1.5)$ & $92(2.2)$ & $139(2.2)$ & 0.0014 \\
\hline Diabetes Type II, EII + EI3 & $490(6.1)$ & $282(6.9)$ & $553(8.6)$ & $<0.0001$ \\
\hline Depression, F32 + F33 & $397(4.9)$ & $221(5.4)$ & $378(5.9)$ & 0.0079 \\
\hline Anxiety, F40 + F4I & $270(3.3)$ & $|7|(4.2)$ & $275(4.3)$ & 0.0023 \\
\hline Osteoporosis, M80 + M8I & $140(1.7)$ & $87(2.1)$ & $194(3.0)$ & $<0.0001$ \\
\hline Fractures, S2 & $457(5.6)$ & $259(6.3)$ & $46 I(7.2)$ & 0.0001 \\
\hline Lung Cancer, C34 & $61(0.8)$ & $34(0.8)$ & $91(1.4)$ & $<0.0001$ \\
\hline \multicolumn{5}{|l|}{ Treatment } \\
\hline ICS in any combination & $1422(17.6 \%)$ & $953(23.3 \%)$ & $2208(34.5 \%)$ & $<0.0001$ \\
\hline LABA in any combination & $1115(13.8 \%)$ & $752(18.4 \%)$ & $1817(28.4 \%)$ & $<0.0001$ \\
\hline LAMA in any combination & $716(8.8 \%)$ & $518(12.7 \%)$ & $1143(17.7 \%)$ & $<0.0001$ \\
\hline SABA & $1068(13.2 \%)$ & $714(17.4 \%)$ & I68I (26.3\%) & $<0.0001$ \\
\hline SABA + SAMA & $48(0.6 \%)$ & $27(0.7 \%)$ & $101(1.6 \%)$ & $<0.0001$ \\
\hline SAMA & $228(2.8 \%)$ & 175 (4.3\%) & 440 (6.9\%) & $<0.0001$ \\
\hline \multicolumn{5}{|l|}{ Health care utilization } \\
\hline Number of times at hospital/year, any reason & 0.42 & 0.50 & 0.67 & $<0.0001$ \\
\hline Number of out hospital visits/year & 1.30 & 1.65 & 2.31 & $<0.0001$ \\
\hline Number of contacts to primary care/year ${ }^{\#}$ & 9.28 & 9.81 & 11.02 & $<0.0001$ \\
\hline Patients with overnight stays (\%) & $2287(28.2)$ & $1367(33.4)$ & $2627(41.0)$ & $<0.0001$ \\
\hline Patients with contacts to primary care (\%) & $6091(75.2)$ & $3035(74.2)$ & $4351(68.0)$ & $<0.0001$ \\
\hline
\end{tabular}

Notes: ${ }^{\#}$ Contacts with primary care is any contact including physical visit, phone contact, laboratory visit, electronic contacts to any type of staff physician, nurse and lab technician. Age, gender, co-morbidity, health care utilization and drug treatment at baseline, ie, two years prior to index date. Group assignment is based on annual exacerbations rate two 2 years prior to index date (patients may move between the groups during follow-up). Treatment "in any combination" includes both drugs taken alone and in combination with other drugs, separately and in fixed combinations. Results are presented as $n$ and percent within brackets. P-values indicate differences between the groups.

Abbreviations: ICS, inhaled steroids; LABA, long-acting beta-agonists; LAMA, long-acting antimuscarinics; SABA, short-acting beta-agonists; SAMA, short-acting antimuscarinics. 
Table 2 Lung Function After Bronchodilatation in COPD Patients Stratified by Exacerbation Rate

\begin{tabular}{|c|c|c|c|c|}
\hline \multirow[t]{2}{*}{ Variable } & & \multicolumn{3}{|c|}{ Annual Exacerbation Rate 2 Years Prior to Index Date } \\
\hline & & $\mathbf{0}$ & $\mathbf{I}$ & $\geq \mathbf{2}$ \\
\hline \multirow[t]{2}{*}{$\mathrm{FEV}_{1}$} & Mean $(95 \% \mathrm{Cl})$ & $1.9(1.8,1.9)$ & $1.8(1.7,1.8)$ & $1.7(1.7,1.8)$ \\
\hline & $\mathrm{N}(\%)$ & $285 I(35.2)$ & $1376(33.6)$ & $1809(28.3)$ \\
\hline \multirow[t]{2}{*}{ FVC } & Mean $(95 \% \mathrm{Cl})$ & $2.9(2.8,2.9)$ & $2.7(2.7,2.8)$ & $2.7(2.7,2.7)$ \\
\hline & $\mathrm{N}(\%)$ & $2716(33.6)$ & $1314(32.1)$ & $1725(27.0)$ \\
\hline \multirow[t]{2}{*}{$\mathrm{FEV}_{1} / \mathrm{FCV}$ ratio } & Mean $(95 \% \mathrm{Cl})$ & $0.60(0.59,0.61)$ & $0.60(0.59,0.61)$ & $0.59(0.58,0.60)$ \\
\hline & $\mathrm{N}(\%)$ & $2863(35.4)$ & I39| (34.0) & I846 (28.8) \\
\hline \multirow[t]{2}{*}{$\mathrm{FEV}_{1} \%$ predicted } & Mean $(95 \% \mathrm{Cl})$ & $65.1(64.4,65.8)$ & $63.1(62.1,64.1)$ & $61.4(60.5,62.2)$ \\
\hline & N (\%) & $3307(40.1)$ & I65I (40.3) & $2193(34.4)$ \\
\hline \multirow[t]{2}{*}{ FVC $\%$ predicted } & Mean $(95 \% \mathrm{Cl})$ & $76.7(76.0,77.4)$ & $74.4(73.5,75.4)$ & $73.4(72.5,74.2)$ \\
\hline & N (\%) & $3079(38.0)$ & I537 (37.6) & 2014 (31.5) \\
\hline
\end{tabular}

Notes: Lung function values are the ones closest to (either before or after) index date. $\mathrm{N}$ (\%) indicate the number of patients and (percent of patients in that group) in whom lung function measurement were available.

Abbreviations: $\mathrm{Cl}$, confidence interval; $\mathrm{FEV}_{1}$, forced expiratory volume in one second; FVC, forced vital capacity.

Supplemental Material Tables 1 and 2 . No difference was observed with respect to baseline characteristics and lung function values between COPD patients with and without concurrent asthma.

\section{Exacerbations}

Between $30 \%$ and $40 \%$ patients in the group who experienced 1 exacerbation per year and $60-70 \%$ of the patients in the groups who had 0 and $\geq 2$ exacerbations per year two years prior to index date had the same rate of exacerbations during up to 11 years follow-up period (Figure 1). Hence, most of the patients (approximately two out of three) who did not have exacerbations during two years prior to index date, did not experience exacerbations during the follow-up. Thus $28 \%$ of all COPD patients did not experience exacerbations before index date and during follow-up. Also, between $60 \%$ and $70 \%$ of the patients who experienced $\geq 2$ annual exacerbations during two years prior to index date also had $\geq 2$ annual exacerbations throughout the whole follow-up period of up to 11 years. This means that $22.4 \%$ of all COPD patients had 2 or more annual exacerbations before index date and during follow-up. Patients with one exacerbation during two years prior to index date moved more frequently between the groups (Figure 2).

\section{Mortality}

Of those who died, $24 \%$ had no exacerbation, $22 \%$ had one exacerbation and $54 \%$ had $\geq 2$ exacerbations during their last year of life. Compared to those having no exacerbation, mortality was higher in patients having 1 (HR [95\% CI] 2.19 [2.05-2.35]) and $\geq 2(4.93$ [4.65-5.21]) annual exacerbations at any time during the follow-up. The mortality was also higher in patients with $\geq 2$ exacerbations/year compared to 1 exacerbation/year (2.24 [2.12-2.37]). All these estimates were adjusted for sex, age, cardiovascular diseases, and lung cancer.

Increased exacerbation frequency was almost linearly associated with increased mortality (Figure 3). The cause of death was more often cardiovascular diseases (40.1\%) in those with no exacerbation than in patients who had a history of exacerbations (31.4\%, $\mathrm{p}<0.0001$; data not shown).

\section{Change in Lung Function}

During the follow-up a total of 941 patients underwent $\geq 3$ lung function measurements that fulfilled the quality criteria for calculations of lung function decline over time. The higher the exacerbation rate the faster lung function declined $(\mathrm{p}=0.0078$; Figure 4).

\section{Pharmacological Treatment}

In Figure 5, the percentage of patients treated with respiratory drugs in the three exacerbation groups ie patients with 0,1 or $\geq 2$ exacerbations/year during the pre-index period is shown. The percentage of patients treated with fixed and free combinations of ICS, LABA and LAMA, and patients treated with SABA alone, increased over time in all three groups (Figure 5). For these groups, there was also 


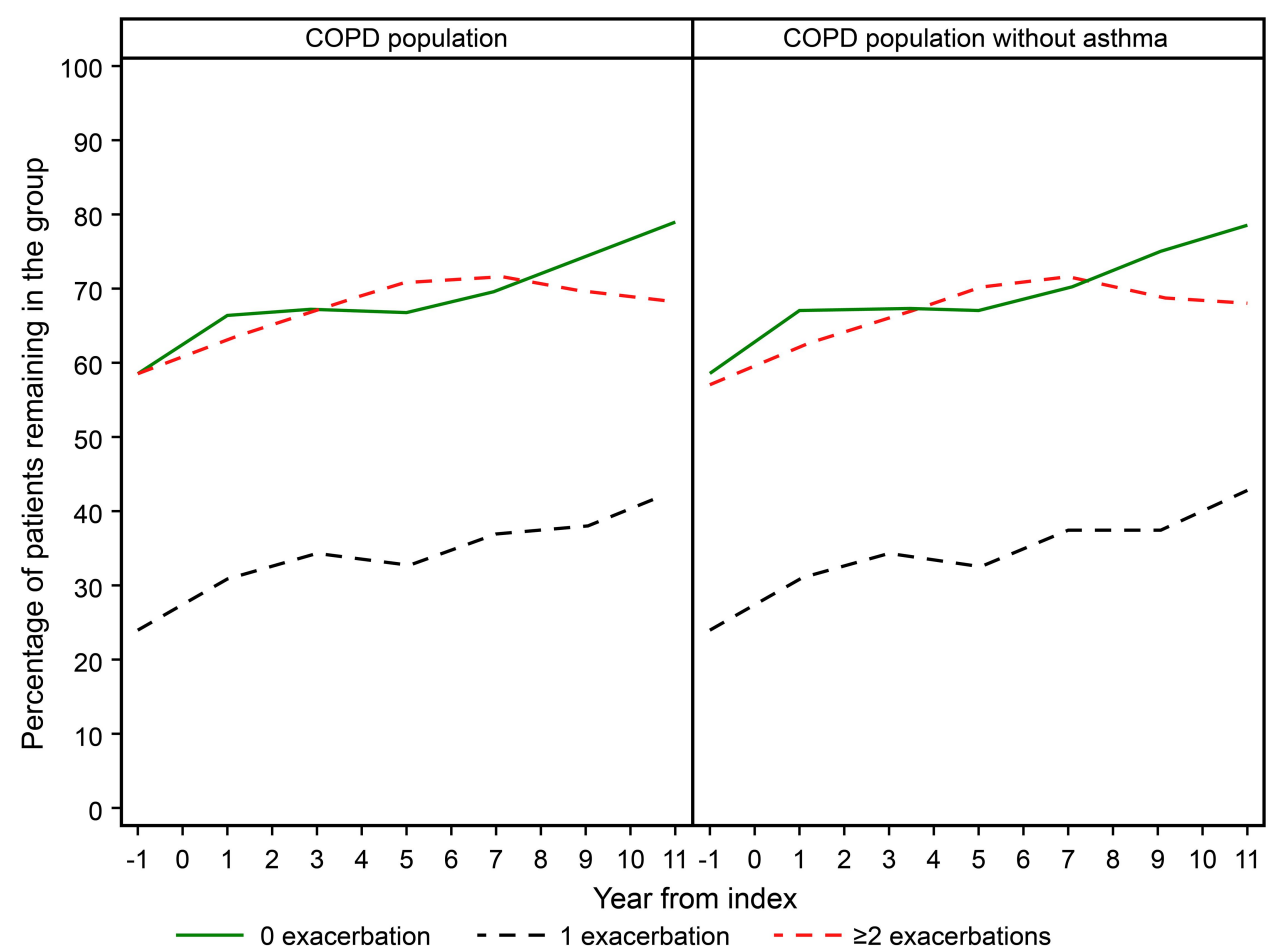

Figure I Exacerbation rate at baseline and during each year of follow-up in COPD patients with $(n=18,586)$ and without ( $n=15,920)$ a concurrent asthma diagnosis. Notes: The percent of patients that remains in the same group as in the previous period of one year is calculated. The most dynamic group is the group with I exacerbation per year, where only around one out of three patients remain in the same group as in the previous one-year period. Around two out of three patients who experienced 0 and $\geq 2$ exacerbations remained in the same group, ie, having 0 or $\geq 2$ annual exacerbations also during follow-up. The pattern is similar in all COPD patients and when COPD without an asthma diagnosis have been deleted.

a higher usage for the given drug for patients with $\geq 2$ exacerbation/year compared to patients with one or no exacerbations. Underlying numbers (data not shown) also suggest higher collection of combination of ICS + LABA + LAMA in patients with $\geq 2$ or 1 exacerbation/year compared to patients with no exacerbations.

\section{Costs}

The total costs (drug costs excluded) showed a peak at year 0 , ie, the year after index date when the diagnosis for COPD was first established. The annual cost during the first year of follow-up was $€ 7108$ per patient in the 0 exacerbation group, $€ 11,981$ per patient in the one exacerbation group, and $€ 20,571$ per patient in the $\geq 2$ exacerbation group, respectively. The higher cost in the groups with frequent exacerbations were mainly driven by hospitalization costs. Total costs were higher in the frequent exacerbation group ( $\geq 2$ exacerbations/year) than in the 0 exacerbation/year group $(\mathrm{p}<0.0001)$ and the 1 exacerbation/year group $(\mathrm{p}<0.0001)$, respectively. If drug costs were excluded, the time trend was stable with no marked total cost changes in the group who experienced $\geq 2$ exacerbations/year whereas there was a slight decrease in the groups with 0 and 1 annual exacerbation (Figure 6A).
The drug costs increased with time after index date in all three groups. Drug costs during the first year of followup were $€ 446$ per patient per year in the 0 exacerbation group, $€ 627$ per patient per year in the 1 exacerbation group and $€ 1049$ per patient per year in the $\geq 2$ exacerbation group. The costs of collected drugs were higher in the frequent exacerbation group ( $\geq 2$ exacerbations/year) compared to the other groups and in the 1 exacerbation group compared with 0 exacerbation $(\mathrm{p}<0.0001$ for all; Figure 6B).

\section{Sensitivity Analysis}

In order to explore the impact of concomitant asthma on the results, a sensitivity analysis was performed by excluding patients with an asthma diagnosis. After exclusion of asthma exacerbation rate, mortality related to exacerbation rate, and lung function decline over time remained unchanged. (Figures 1, 3 and 4).

\section{Discussion}

In this study, it was demonstrated that outcomes such as lung function decline and mortality in COPD are not only related to the presence of exacerbations per se but also that there is 


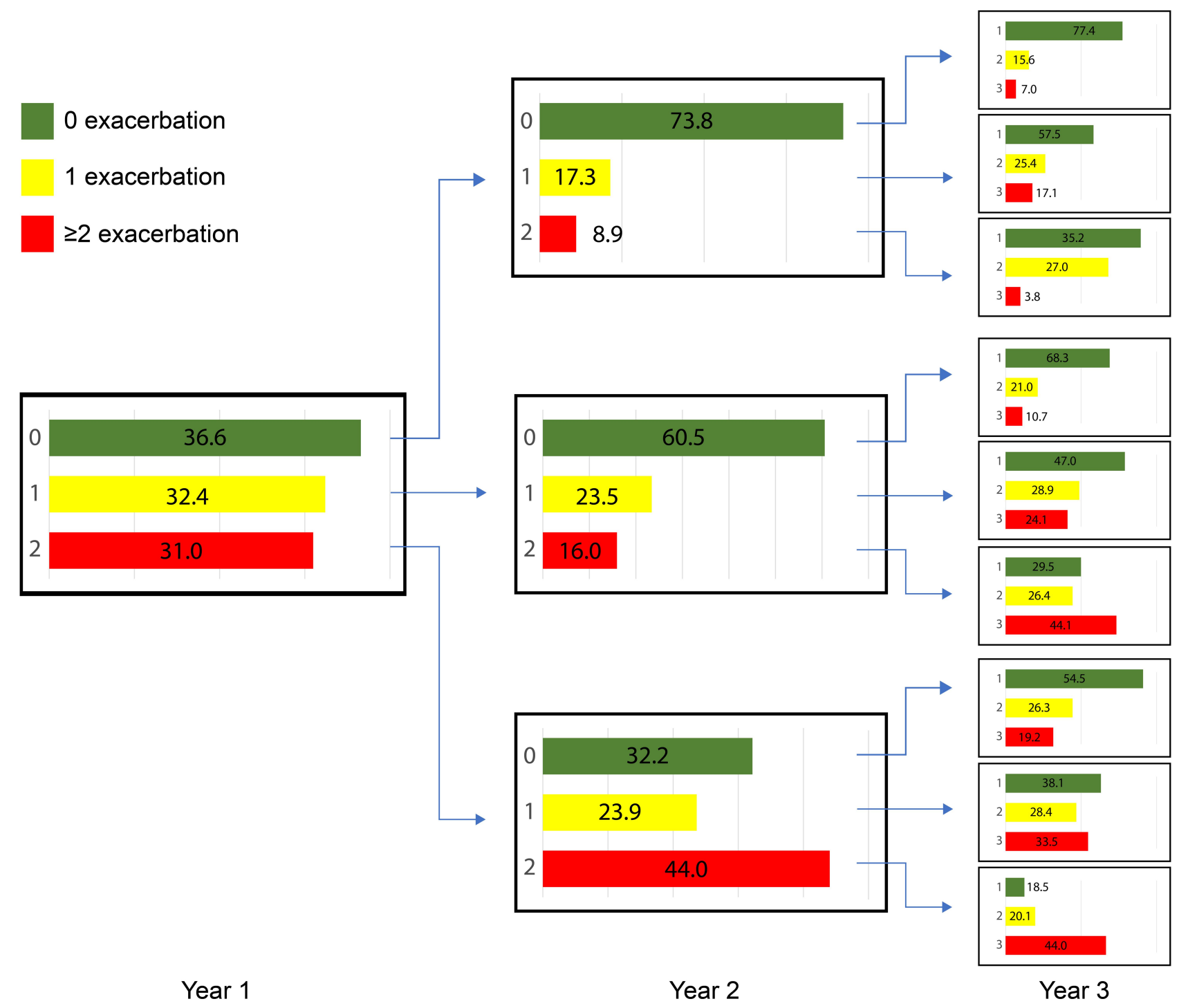

Figure 2 Exacerbation rate during the first three years of follow-up after index date in 18,586 patients with COPD.

Note: The green, yellow and red color bars represent the groups of patients with 0.1 or $\geq 2$ exacerbations during the first, second and third year of follow-up, respectively.

a clear quantitative relationship between those outcomes and exacerbation rate, the higher number of exacerbations, starting below one exacerbation per year, the more rapid lung function decline and the higher death rate. Furthermore, it was shown that the tendency to experience exacerbations over time is rather a constant phenomenon in a patient with COPD. Approximately two of three patients who had no history of exacerbations did not experience any exacerbation during the follow-up and about the same amount of patients (60-70\%) who had experienced $\geq 2$ exacerbations per year during the preindex period continued to have $\geq 2$ exacerbations during the follow-up period. However, we observed a greater variability in exacerbation rate during the follow-up among patients with a history of one exacerbation per year.
In the GOLD document, group A and B are regarded as the low-risk groups as they have only one exacerbation in the last year. In the present study, it was clearly demonstrated that all outcomes were related to exacerbation rate and that patients with one exacerbation per year were worse off than patients who did not experience any exacerbations. This may have implication for treatment recommendations as there seems to be a clear indication for including patients with one annual exacerbation in the high-risk groups, ie, group $\mathrm{C}$ and $\mathrm{D}$. Our data suggest that an exacerbation rate of one per year constitutes a risk factor for negative outcomes.

Our results are in agreement with earlier studies that have shown that a history of frequent exacerbations is 


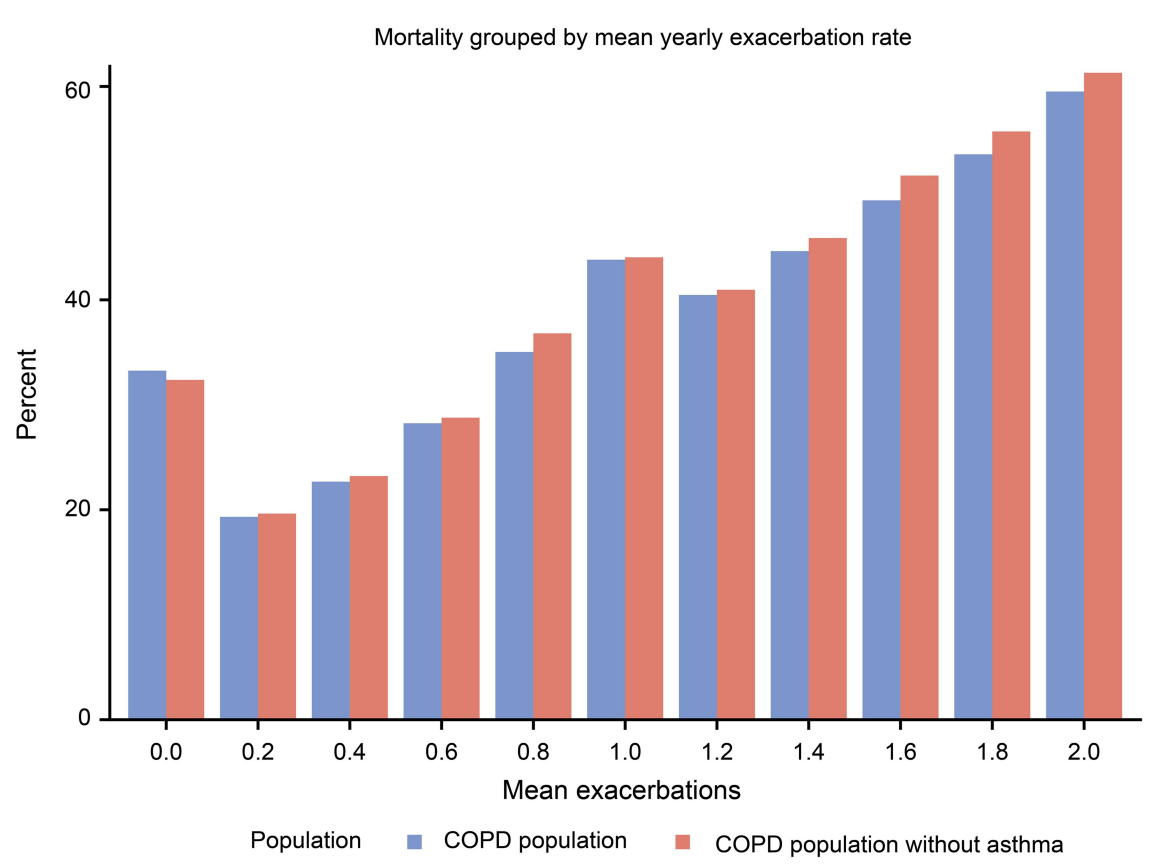

Figure 3 Mortality related to the average number of annual exacerbations during the complete follow-up period before death in COPD patients with ( $\mathrm{n}=18,586)$ and without $(n=15,920)$ a concurrent asthma diagnosis.

Post bronchodilator lung function decline related to exacerbation rate during follow up

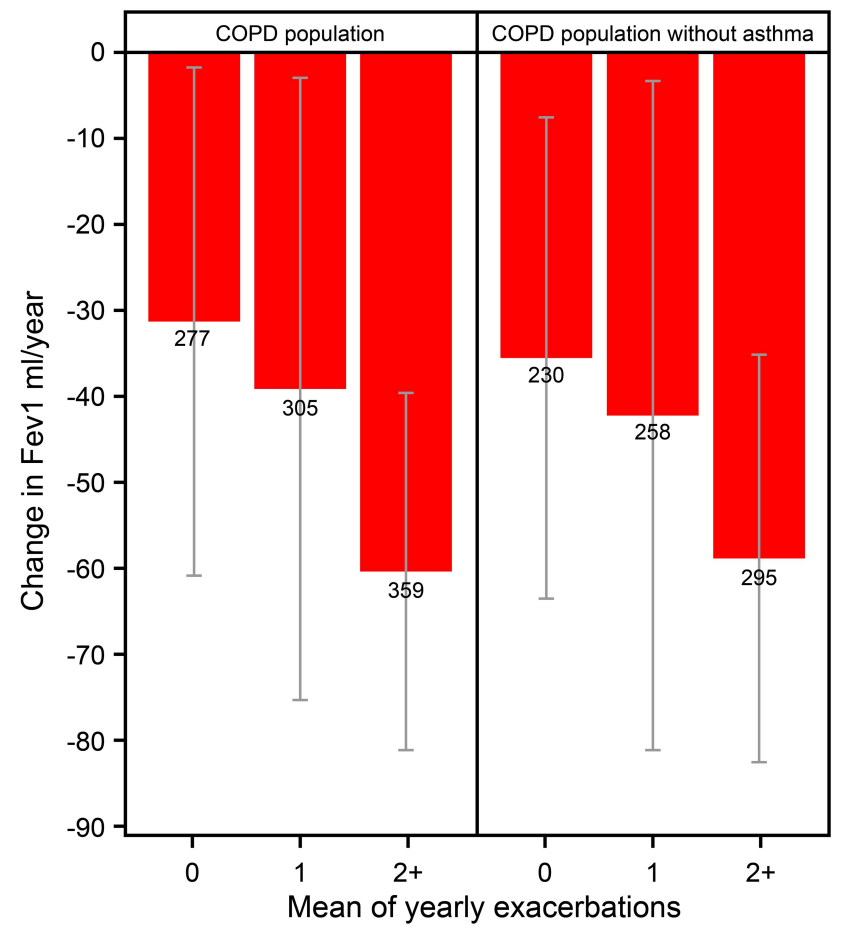

Figure 4 Post bronchodilator lung function decline related to exacerbation rate during follow-up in COPD patients with $(n=18,586)$ and without $(n=15,920)$ a concurrent asthma diagnosis.

Notes: Data are given for patients who had experienced, on average, 0 , I, or $\geq 2$ exacerbations per year during follow-up. Lung function $\left(\mathrm{FEV}_{\mathrm{I}}\right)$ decline is based on three or more measurements and given as post-bronchodilator $\mathrm{FEV}_{1}$ decline in $\mathrm{mL}$ / year. Mean values and $95 \%$ confidence interval.

Abbreviation: $\mathrm{FEV}_{\mathrm{l}}$, forced expiratory volume in one second. a predictor of future exacerbations. ${ }^{4}$ In the present study, it was shown that over $40 \%$ of the patients with a physician diagnosed COPD did not have exacerbations, neither during pre-index nor during follow-up. This finding emphasizes the importance of the exacerbation history when decisions about pharmacological and nonpharmacological treatment is taken, in particular when treatment is commenced in previously untreated COPD patients. Our findings confirm previous observations that frequent exacerbations among COPD patients are associated with increased mortality. ${ }^{2,411}$ We observed almost a linear relationship between exacerbation frequency and mortality. This, again, implies that a careful exacerbation history is imperative when optimizing the treatment for patients who have a history of exacerbations in order to likely bring down mortality in this group of COPD patients.

It has been shown that addition of ICS to bronchodilators provides better efficacy in the subset of COPD patients with frequent exacerbations and with higher blood eosinophil count $(\geq 300$ cells $/ \mu \mathrm{L})$ and/or a history of asthma than therapy with bronchodilators. ${ }^{12-14}$ Our study results showed that treatment with bronchodilators and inhaled steroids is more common in patients with frequent exacerbations than in patients with no exacerbations which would be according to the guidelines at the 


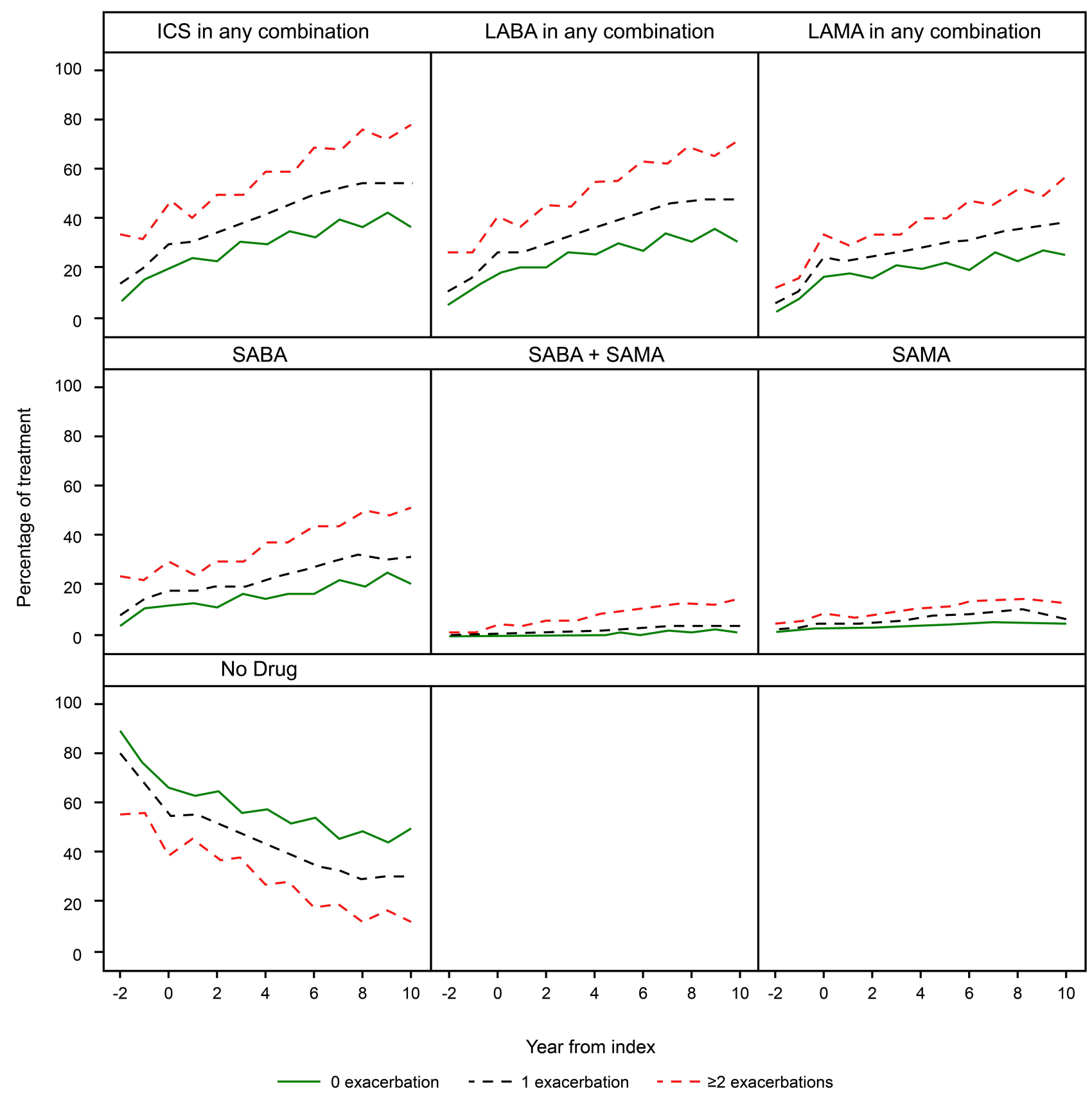

Figure 5 Cumulative dispensation, representing prescription during the preceding year, of respiratory drugs during a follow-up period of 8 years in 18,586 patients with COPD.

Abbreviations: ICS, inhaled steroids; LABA, long-acting beta-agonists; LAMA, long-acting antimuscarinics; SABA, short-acting beta-agonists; SAMA, short-acting antimuscarinics.

time of the study and to current guidelines. From these data, it could be concluded that a substantial number of patients who do not experience exacerbations are treated with ICS which, however, is not in agreement with Swedish or international guidelines. ${ }^{2,15,16}$ According to Swedish (and international) guidelines long-acting bronchodilators constitute first line for symptom relief in COPD. The obvious difference in prescription of bronchodilators between patients with no exacerbations compared with frequent exacerbations may indicates that patients who do not experience exacerbations are under-treated with bronchodilators. Apart from a possible over-prescription of ICS in patients who do not experience exacerbations, our findings emphasize the importance of a careful assessment of the clinical picture in order to prescribe bronchodilators to COPD patients who need symptom relief. 


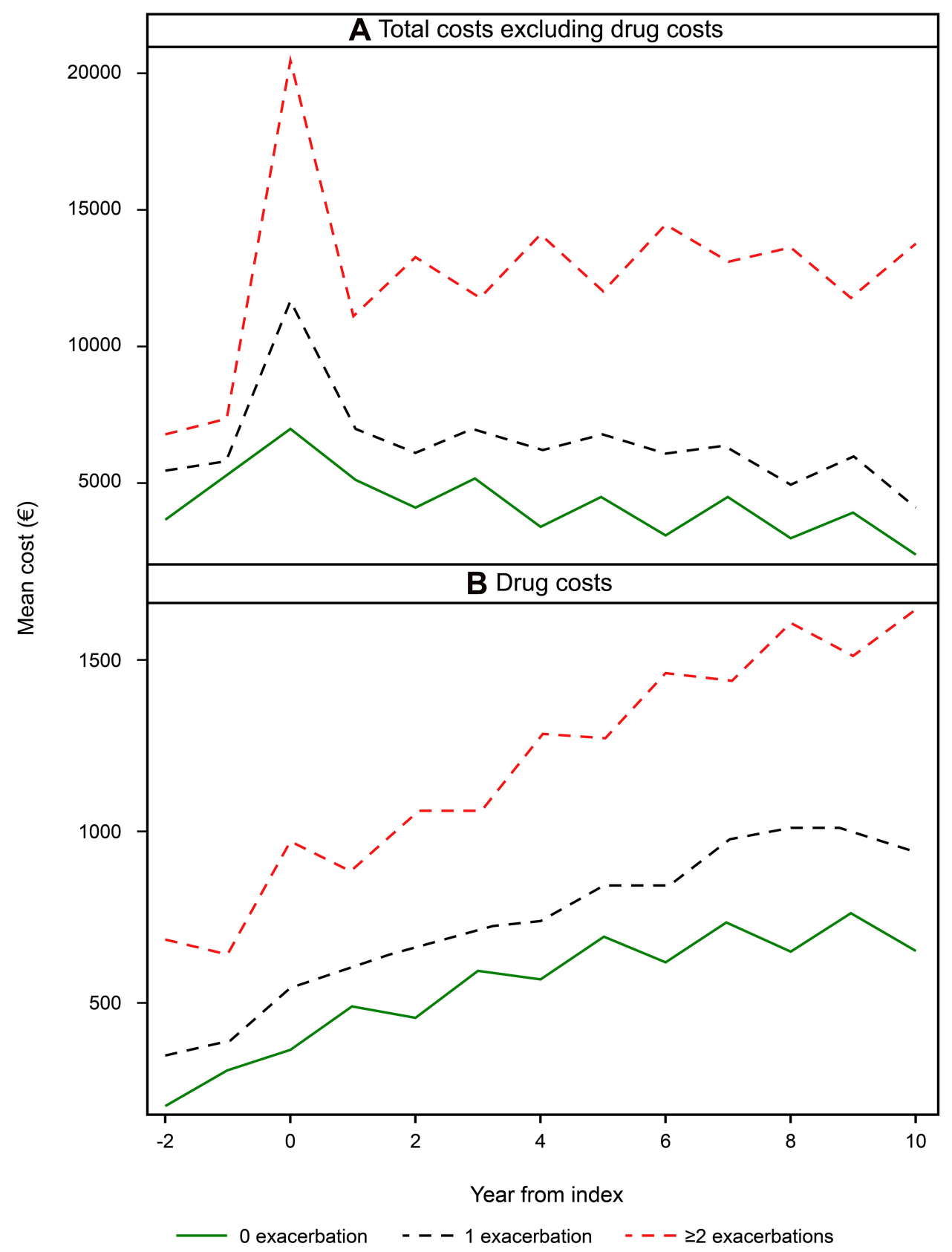

Figure 6 Total direct costs excluding drug costs $(\mathbf{A})$ and costs for drugs in COPD patients (B) with 0 , I and $\geq 2$ exacerbations per year during II years of follow-up.

We observed that exacerbations drive lung function impairment over time and these findings are in line with previous results. In the classical study by Fletcher and colleagues, it was reported that in healthy non-smoking adults had an $\mathrm{FEV}_{1}$ decline of, on average, $30 \mathrm{~mL}$ per year. ${ }^{17}$ In the present study, the annual decline in $\mathrm{FEV}_{1}$ was approximately $30 \mathrm{~mL}$ in COPD patients who did not experience exacerbations prior to the index date or during follow-up. This is in line with the findings by Kesten et al who did not show a substantial increase in lung function decline in the COPD with the lowest exacerbation rate. ${ }^{18}$ In a small prospective study, a somewhat faster lung function decline was observed in patients with frequent ( $>2.92$ exacerbations/year) than in patients with less frequent $\left(<2.92\right.$ per year) exacerbations. ${ }^{19}$ In the present study, it was confirmed that lung function decline is related to exacerbations and that this relationship seems to be quantitative, the more frequent exacerbations the faster lung function decline.

We also observed that the direct healthcare costs incurred by COPD patients with frequent exacerbations were higher 
than COPD patients with no history of exacerbations, which supported findings from other studies. ${ }^{20,21}$ Furthermore, the drug costs increased with time whereas the other direct healthcare costs did not change much over time. During the followup of the present study (2000-2014), the awareness of COPD and how to manage the disease improved substantially in Sweden, ${ }^{22,23}$ which could explain that the drug costs increased whereas other costs were kept down due to improved management. During the first year of follow-up, the total costs, but not the drug costs, were high, a finding that was most clear in the group with $\geq 2$ annual exacerbations. These costs were associated with measures related to COPD diagnosis and to hospitalizations.

In general, the patients with a dual diagnosis of asthma and COPD are younger with a higher clinical burden than those diagnosed with COPD alone. ${ }^{24,25}$ This is in agreement with the finding of the present study in which the prevalence of comorbidities was generally somewhat higher in the whole group compared to the group with pure COPD, ie, when asthma was excluded. No difference was observed with respect to age among patients with both asthma and COPD and patients with COPD alone suggesting that the COPD diagnosed may have been established at later stage in patients already having an asthma diagnosis. This interpretation is in line with a previous ARCTIC study in which asthma was more common in COPD patients receiving their COPD diagnosis at a late stage indicating that the asthma diagnosis may delay the diagnosis of COPD. ${ }^{26}$ Although patients with both asthma and COPD seem to be slightly worse off with regard to disease burden, exacerbation rate, lung function decline and mortality rate did not differ between the groups indicating that a concurrent asthma diagnosis did not have an impact on these outcomes which may be attributed to the fact that study patients had a moderate or mild COPD.

The present study has several important strengths. The real-world study design, long follow-up period and the large sample size of high-quality national registry data from primary care settings adequately reflect the general population and clinical practice in Sweden. Additionally, the study carried out a comprehensive assessment (2000-2014) of history of exacerbation and its association with the exacerbation rate, mortality, COPD treatments, lung function and healthcare costs during the long follow-up time. Data quantification in this study can allow decision makers to understand the impact of exacerbations frequency in COPD patients, which may serve as the basis for the development of treatment strategies to achieve better outcomes in millions of patients with COPD worldwide.
Nevertheless, this study also has certain limitations. The retrospective study design introduces the potential for bias and confounding due to variables that may not have been accounted for in our analysis. Although all patients had physician-diagnosed COPD, the accuracy of COPD diagnoses and the severity of disease could not be verified in all patients, as spirometry data were missing in a number of patients. Only direct costs were included in the study and no data was available on smoking history of the included patients. Furthermore, the study was conducted only in Swedish patients; it is therefore uncertain whether these findings can be extrapolated to a more diverse group of patients and to other healthcare systems.

\section{Conclusion}

In conclusion, our findings demonstrated that COPD patients with frequent exacerbations have a higher risk of future exacerbations, mortality, rapid lung function decline and incur greater healthcare costs compared with infrequent exacerbators and non-exacerbators, demonstrating a significant economic burden. Our results clearly indicate that a concomitant asthma diagnosis does not have an impact on the outcomes in patients with COPD in a Swedish primary care setting. Management of COPD directed to reduce incidence of exacerbations improves clinical and economic outcomes in COPD patients.

\section{Key Messages}

What is the key question?

- Is exacerbation frequency associated with clinical and economic impact in Swedish COPD patients?

What is the bottom-line?

- COPD patients with exacerbations have a higher risk of future exacerbations, mortality, rapid lung function decline and incur greater healthcare costs compared with infrequent exacerbators and, even more, nonexacerbators, demonstrating a significant economic burden.

Why read on?

- The relationship between exacerbation rate and mortality increased from levels below one annual exacerbation and was almost linear. This implies that having one exacerbation per year increased the risk of future 
exacerbations and death, a finding that may question the classification of the GOLD A and B groups as low-risk groups.

\section{Data Sharing Statement}

The data for this study were obtained from electronic medical records in primary care and the Swedish National Health Register. Restrictions apply to the availability of these data, which were used under license for the current study, and so are not publicly available. Data are however available from IQVIA Solutions, Solna, Sweden, upon reasonable request and with permission of the Swedish National Health Register.

\section{Acknowledgments}

The authors would like to thank Harneet Kaur (Novartis) for managing and providing writing assistance in the development of this manuscript.

\section{Author Contributions}

All authors contributed to data analysis, drafting or revising the article, have agreed on the journal to which the article will be submitted, gave final approval of the version to be published, and agree to be accountable for all aspects of the work.

\section{Funding}

This study was funded by Novartis Pharma AG, Basel, Switzerland.

\section{Disclosure}

Kjell Larsson has, during the last 5 years, on one or more occasion served in an advisory board, served as a speaker and/or participated in education activities arranged by AstraZeneca, Boehringer Ingelheim, GlaxoSmithKline, Chiesi, Sanofi, Novartis, Orion, and Teva. Christer Janson reports personal fees from AstraZeneca, Boehringer Ingelheim, Chiesi, GlaxoSmithKline, Novartis, and Teva, outside the submitted work. Karin Lisspers reports personal fees from AstraZeneca, Novartis, Boehringer-Ingelheim, Glaxo-Smith-Kline, and Chiesi, outside the submitted work. Björn Ställberg reports personal fees from AstraZeneca, Novartis, Boehringer Ingelheim, GlaxoSmithKleine Meda, Teva, and Chiesi, outside the submitted work. Gunnar Johansson has participated in the steering committee organized by Novartis for this study and served on advisory boards arranged by AstraZeneca, Novo Nordisk and Takeda. Florian
S Gutzwiller and Karen Mezzi are employees of Novartis Pharma AG. Bine Kjoeller Bjerregaard and Leif Jorgensen are employees of IQVIA, and received remuneration from Novartis in relation to statistical analyses. The authors report no other conflicts of interest in this work.

\section{References}

1. Lozano R, Naghavi M, Foreman K, et al. Global and regional mortality from 235 causes of death for 20 age groups in 1990 and 2010: a systematic analysis for the Global Burden of Disease Study 2010. Lancet. 2012;380(9859):2095-2128. doi:10.1016/S0140-6736(12) 61728-0

2. Global Initiative for Chronic Obstructive Lung Disease (GOLD). Global strategy for the diagnosis, management, and prevention of chronic obstructive pulmonary disease [updated 2019]; 2019.

3. Blasi F, Cesana G, Conti S, et al. The clinical and economic impact of exacerbations of chronic obstructive pulmonary disease: a cohort of hospitalized patients. PLoS One. 2014;9(6):e101228. doi:10.1371/ journal.pone. 0101228

4. Hurst JR, Vestbo J, Anzueto A, et al. Susceptibility to exacerbation in chronic obstructive pulmonary disease. $N$ Engl J Med. 2010;363 (12):1128-1138. doi:10.1056/NEJMoa0909883

5. Celli BR, MacNee W, Force AET. Standards for the diagnosis and treatment of patients with COPD: a summary of the ATS/ERS position paper. Eur Respir J. 2004;23(6):932-946. doi:10.1183/ 09031936.04.00014304

6. Dal Negro R. Optimizing economic outcomes in the management of COPD. Int $J$ Chron Obstruct Pulmon Dis. 2008;3(1):1-10. doi:10.2147/COPD.S671

7. Dalal AA, Christensen L, Liu F, et al. Direct costs of chronic obstructive pulmonary disease among managed care patients. Int J Chron Obstruct Pulmon Dis. 2010;5:341-349. doi:10.2147/ COPD.S13771

8. Socialstyrelsen (The Swedish National Board of Health and Welfare); 2017. Available from: http://www.socialstyrelsen.se/register. Accessed January 07, 2017.

9. Toy EL, Gallagher KF, Stanley EL, et al. The economic impact of exacerbations of chronic obstructive pulmonary disease and exacerbation definition: a review. COPD. 2010;7(3):214-228. doi:10.3109/ 15412555.2010.481697

10. Statistics Sweden [webpage on the Internet]. Longitudinal Integration Database for Health Insurance and Labour Market Studies [LISA by Swedish acronym 2004].Available from: https://www.scb.se/en/ser vices/guidance-for-researchers-and-universities /SSLidfhialmsLbSaAa.v-m-fl-rl-i-f-h-i-a-l-s-lLaJ. Accessed March 05, 2021

11. Santibanez M, Garrastazu R, Ruiz-Nunez M, et al. Predictors of hospitalized exacerbations and mortality in chronic obstructive pulmonary disease. PLoS One. 2016;11(6):e0158727. doi:10.1371/journal.pone. 0158727

12. Bafadhel M, Peterson S, De Blas MA, et al. Predictors of exacerbation risk and response to budesonide in patients with chronic obstructive pulmonary disease: a post-hoc analysis of three randomised trials. Lancet Respir Med. 2018;6(2):117-126. doi:10.1016/S22132600(18)30006-7

13. Pascoe S, Barnes N, Brusselle G, et al. Blood eosinophils and treatment response with triple and dual combination therapy in chronic obstructive pulmonary disease: analysis of the IMPACT trial. Lancet Respir Med. 2019;7(9):745-756. doi:10.1016/S22132600(19)30190-0

14. Vogelmeier CF, Kostikas K, Fang J, et al. Evaluation of exacerbations and blood eosinophils in UK and US COPD populations. Respir Res. 2019;20(1):178. doi:10.1186/s12931-019-1130-y 
15. Läkemedelverkets Medical Products Agency (Sweden): Läkemedelverkets expert panel. [Farmakologisk behandling av kroniskt obstruktiv lungsjukdom (KOL)-behandlingsrekommendationer]. Available from: https://www.lakemedelsverket.se/sv. SMALKolKb. Accessed March 5, 2021. Swedish.

16. Sulku J, Janson C, Melhus H, et al. A cross-sectional study assessing appropriateness of inhaled corticosteroid treatment in primary and secondary care patients with COPD In Sweden. Int J Chron Obstruct Pulmon Dis. 2019;14:2451-2460. doi:10.2147/COPD.S218747

17. Fletcher C, Peto R. The natural history of chronic airflow obstruction. Br Med J. 1977;1(6077):1645-1648. doi:10.1136/bmj.1.6077.1645

18. Kesten S, Celli B, Decramer M, et al. Adverse health consequences in COPD patients with rapid decline in FEV1-evidence from the UPLIFT trial. Respir Res. 2011;12:129. doi:10.1186/ 1465-9921-12-129

19. Donaldson GC, Seemungal TA, Bhowmik A, et al. Relationship between exacerbation frequency and lung function decline in chronic obstructive pulmonary disease. Thorax. 2002;57(10):847-852. doi:10.1136/thorax.57.10.847

20. Dalal AA, Patel J, D'Souza A, et al. Impact of COPD exacerbation frequency on costs for a managed care population. J Manag Care Spec Pharm. 2015;21(7):575-583. doi:10.18553/jmcp.2015.21.7.575
21. Patel JG, Coutinho AD, Lunacsek OE, et al. COPD affects worker productivity and health care costs. Int J Chron Obstruct Pulmon Dis. 2018;13:2301-2311. doi:10.2147/COPD.S163795

22. Lisspers K, Johansson G, Jansson C, et al. Improvement in COPD management by access to asthma/COPD clinics in primary care: data from the observational PATHOS study. Respir Med. 2014;108 (9):1345-1354. doi:10.1016/j.rmed.2014.06.002

23. Stallberg B, Janson C, Johansson G, et al. Management, morbidity and mortality of COPD during an 11-year period: an observational retrospective epidemiological register study in Sweden (PATHOS). Prim Care Respir J. 2014;23(1):38-45. doi:10.4104/pcrj.2013.00106

24. Gibson PG, McDonald VM. Asthma-COPD overlap 2015: now we are six. Thorax. 2015;70(7):683-691. doi:10.1136/thoraxjnl-2014206740

25. Kumbhare S, Pleasants R, Ohar JA, et al. Characteristics and Prevalence of Asthma/Chronic Obstructive Pulmonary Disease Overlap in the United States. Ann Am Thorac Soc. 2016;13 (6):803-810. doi:10.1513/AnnalsATS.201508-554OC

26. Larsson K, Janson C, Stallberg B, et al. Impact of COPD diagnosis timing on clinical and economic outcomes: the ARCTIC observational cohort study. Int $J$ Chron Obstruct Pulmon Dis. 2019;14:995-1008. doi:10.2147/COPD.S195382

\section{Publish your work in this journal}

The International Journal of COPD is an international, peer-reviewed journal of therapeutics and pharmacology focusing on concise rapid reporting of clinical studies and reviews in COPD. Special focus is given to the pathophysiological processes underlying the disease, intervention programs, patient focused education, and self management protocols. This journal is indexed on PubMed Central, MedLine and CAS. The manuscript management system is completely online and includes a very quick and fair peer-review system, which is all easy to use. Visit http://www.dovepress.com/testimonials.php to read real quotes from published authors. 